\title{
Study on the Application of Mildew Inhibitor in Military Warehouse Equipment Storage
}

\author{
Li Li $^{1 *}$, Zhao Hongqiang ${ }^{1}$, Sun weiqi ${ }^{1}$, Ren $\mathrm{Jian}^{1}$, Zhou Wei ${ }^{1}$, Li Kun ${ }^{1}$, Wang Xiaoyan ${ }^{1}$ \\ ${ }^{1}$ Naval Aviation University Qingdao Branch, Qingdao, China, 266041
}

\begin{abstract}
Firstly, the basic conditions of the fungicide were introduced, its action principle was analyzed, the use method of the fungicide in the military warehouse was put forward, and the factors influencing the application effect were discussed.
\end{abstract}

\section{Introduction}

Some military warehouses are built in coastal areas. Due to the humidity and rain in these areas and the high relative humidity in the warehouse, the equipment has a certain degree of mildew phenomenon, which not only reduces the storage quality of equipment, but also causes the phenomenon of equipment scrapping. The use of fungicides can greatly reduce the loss caused by mildew, so it has a wide application prospect in the anti mildew work of military warehouse equipment.

\section{Basic conditions of fungicides}

Mold inhibitor is a kind of polymer material additive which can inhibit the growth of mold and kill mold. It can protect warehouse equipment from mold erosion and maintain good appearance and physical and mechanical properties. Due to the special environment of military warehouse, the fungicide that can be selected must meet the following conditions ${ }^{[1-2]}$ :

(1) High efficiency and low toxicity not only have good antibacterial effect, but also do not harm health, and pollute the environment.

(2) It has a wide range of application, that is, it can kill or inhibit a variety of fungi.

(3) Good solubility, or good water solubility, or organic solvent solubility, it is best to meet different requirements at the same time.

(4) It is stable in nature, not easy to volatilize, not easy to react due to light, heat, acid and other physical and chemical factors, and has a long effective time.

(5) No chemical reaction with the components in the equipment will weaken the effectiveness of the agent, and the performance and quality of the warehouse equipment can not be affected.

\section{Action principle of fungicide}

Usually, many characteristics of fungi have changed after being treated with fungicides, such as growth, reproduction, spore germination, cell mitosis and other physiological, biochemical reactions and metabolic activities will be affected. Therefore, the growth and reproduction of fungi will be inhibited, and finally the purpose of killing fungi will be achieved ${ }^{[3-7]}$.

\subsection{Destroy Cell Structure}

\section{(1) Cell Wall Damage}

Fungal cell wall is composed of mannan, dextran, protein, cellulose and chitin. Some antibacterial substances can competitively inhibit the enzyme reaction to synthesize the components of cell wall, thus inhibiting the synthesis of fungal cell wall.

(2) Destruction of Cell Membrane

The main physiological function of cell membrane is to control the transportation and exchange of nutrients and metabolic wastes inside and outside the cell. Once the cell membrane is destroyed, a large amount of water in the cell will be lost, and various ions, enzymes and intermediate products will seep out of the cell, making the cell autolysis, thus inhibiting the growth and reproduction of moldy rot microorganisms and achieving the purpose of inhibiting or killing fungi.

(3) Destroy the Structure of Proteins

Without protein, there would be no life activity. Some fungicides can decompose protein and destroy the lipophilic bond between protein chains. If the protein structure is destroyed, the life activities of fungi will be inhibited and the cells will die.

\subsection{Affect Metabolism}

(1) Inhibition of Enzyme Activity 
One of the basic characteristics of fungi is metabolism. These metabolic activities all need a series of complex chemical changes in the organism. These chemical changes are carried out rapidly under mild conditions and catalyzed by enzymes. It can speed up the chemical reaction in organism and make the reaction change in a certain order. In a word, if the structure or activity of enzyme is destroyed, the growth and reproduction of fungi will be inhibited or even die.

(2) Respiratory Inhibition

The respiration of most fungi is to absorb oxygen and release carbon dioxide, and release the energy needed for life activities. Any link of energy metabolism may inhibit the growth and reproduction of fungi.

\subsection{Affect Fungal Reproduction}

Chromosome is one of the main components of nucleus. It is mainly composed of DNA and protein. DNA is the main material basis of heredity, and its main physiological function is to transmit genetic information. Fungicides can cause chromosome breakage and recombination, or cause chromosome aberration, or cause base substitution, thus resulting in chromosome mutation. In addition, fungicides can also react with DNA so that chromosomes cannot be separated during mitosis, or even if they do, they hinder the formation of spindles and prevent daughter nuclei from being pulled to new cells.

\section{Method of application ${ }^{[8-9]}$}

\subsection{Addition Method}

This method is to add a certain proportion of mold inhibitor in the manufacturing process of materials or products. It can be added with raw materials at the same time, can also be added in a certain part of the way, or even added in the finished product. Powder can be added, or the agent dissolved in water or other organic solvents can be added.

\subsection{Impregnation Method}

Immerse the material or product in a certain concentration of mildew inhibitor for a certain period of time, and then take it out to dry or dry, so that the surface and interior of the material or product can be adsorbed to the mold inhibitor. According to the different processing objects, it is necessary to master the time and temperature of impregnation.

\subsection{Coating Method}

A certain concentration of anti mildew agent is coated on the surface of materials or products with brushing tools (brush etc.), and the coating position is not only the outer surface, but also the inner surface.

\subsection{Spray Method}

Spraying a certain concentration of mold inhibitor on the surface of materials or products with a sprayer is also best related to the inner surface. Spray must be uniform and droplets should not be too large. In order to ensure the sterilization effect, the spray should be closed for a period of time before entering the warehouse.

\subsection{Fumigation}

The powder or tablet of base volatile fungicide with the base domain between 15 and 19 is placed in the space of the product or container, and is allowed to volatilize, and gradually emit bactericidal gas. The gas is spread in the confined space to play an anti mildew role.

\section{Judgment of the efficacy of fungicides [10]}

The effectiveness of the so-called fungicide refers to the minimum concentration that can kill or inhibit the growth and development of mold and other microorganisms. It is generally expressed by the minimum inhibitory concentration of drugs on microorganisms.

Several simple and practical determination methods are introduced below.

\subsection{Plate Smearing Method}

This method is to the culture medium and chemicals are evenly mixed in the culture dish. After the medium is solidified, the spores of $10 \sim 15$ kinds of test bacteria can be inoculated radially on the plate, and then cultured in the incubator for a period of time. Finally, the minimum inhibitory concentration of various fungi is determined according to their development.

\subsection{Filter Paper Inhibition Circle Method}

This method is to inject the culture medium mixed with experimental bacteria into the culture dish and make it into a plate, stick the filter paper with different concentrations of medicine on the plate, culture at a certain temperature, and determine according to the presence of transparent inhibition circle.

\subsection{The Liquid Oscillation Culture Method}

This method is to adding different concentrations of medicament, culture medium and spore suspension into the test tube, and shaking culture at a certain temperature can determine whether the fungicide has inhibitory effect on certain microorganisms by the growth of mycelium and spore (mold, etc.). 


\section{Factors affecting the effect of fungicides ${ }^{[11-14]}$}

\subsection{The Influence of Mold Rot Microorganism}

(1) The effect of fungicides is affected by the different types of moldy microorganisms in equipment pollution Some fungicides are particularly effective in killing or inhibiting yeasts, some are particularly effective in killing or inhibiting yeasts, some are effective against both, and some are effective against specific bacteria. Therefore, what kind of microorganisms or bacteria are contaminated by the equipment will affect the effect of the fungicide.

(2) The influence of different degrees of equipment contaminated with moldy rot microorganisms on the anti mildew effect

The time for all kinds of fungicides to completely kill moldy rot microorganisms is related to the number of microorganisms per unit volume. The more the number of moldy rot microorganisms per unit volume, the longer the sterilization time.

\subsection{Effect of Fungicides}

(1) The structure and physicochemical properties of the fungicide affect the antibacterial effect

Due to the different chemical structures of various fungicides, their bactericidal effects on various microorganisms are also different. For example, some fungicides have good fungicidal effect, while others have better killing effect on bacteria.

(2) The solubility and dispersion of fungicides determine their germicidal efficacy

Different fungicides have different solubility and dispersibility. Some of them are dissolved in water, while others are dissolved in ethanol or other organic solvents. Therefore, different adding methods should be adopted for different fungicides.

(3) The concentration of fungicide affects the effect of mildew prevention

The more concentrated the fungicide added to the equipment is not the better, some fungicides in a certain concentration range, with the increase of concentration, the bactericidal effect will be improved, but if the concentration is too high, the sterilization effect will be reduced, such as ethanol. The reason is that too high concentration can coagulate the protein on the surface or around the cell membrane, which affects the ability of the fungicide to penetrate into the microbial body.

(4) The mixed use of fungicides can affect the antibacterial effect

Each fungicide has its own scope of action. When two or more fungicides are used together, they can play a synergistic role. It can be a mixture of the same type of fungicides or different types of fungicides, so as to improve the antibacterial effect.

\subsection{Influence of External Environment and Equipment Itself}

(1) Temperature has great influence on germicidal efficacy

Since each kind of fungicide has its own decomposition temperature, the heating temperature of the equipment added with the mold inhibitor should not exceed the decomposition temperature of the fungicide in the production process, otherwise the fungicide will decompose and become invalid.

(2) Effect of $\mathrm{pH}$ value on mildew prevention

Each fungicide has its own suitable $\mathrm{pH}$ range. The ionization degree of alkali decreases with the increase of $\mathrm{pH}$ and increases with the decrease of $\mathrm{pH}$.

(3) Humidity often affects the germicidal efficacy of gas fungicides

The effect of some gaseous fungicides is affected by moisture (humidity). Generally, the higher the humidity, the worse the sterilization effect.

(4) The ingredients in the equipment may reduce the mildew resistance

(1) Physical effect, because a certain component in the equipment adsorbs the fungicide, or its role is just opposite to that of the fungicide, thus weakening the effect of the fungicide.

(2) Chemical action, that is, the chemical reaction between the fungicide and a certain component in the equipment will lead to the reduction or loss of the function of the fungicide. Such as ammonia on formaldehyde, metal salts on sulfur compounds, etc., have antagonistic effect.

\section{Conclusion}

Because the military warehouse only through ventilation, dehumidification, sealing and other environmental conditions control can not fully meet the requirements of equipment storage, the use of mold inhibitor can effectively alleviate the loss of equipment performance caused by mold. The development trend of fungicides in the future is to develop compound fungicides, improve their fungicidal properties, overcome their resistance, extend their validity period, and ensure their high efficiency, low toxicity, safety, broad spectrum and wide range of application.

\section{Reference}

1. Xiao Liping, Li Linsheng. Antimicrobial preservatives (I) historical definition and classification of antimicrobial preservatives [J]. Daily chemical industry, 2001,5.

2. Zhang Tianen, Zhang Yibin. Handbook of fungicides [M]. Shanghai: Shanghai Science and Technology Literature Publishing House, 1993, 3:5-16.

3. Wang Kang. Inhibition effect and mechanism of safety and environmental protection mold inhibitor on animal specimen mold [D]. Guangdong Ocean University, 2017. 
4. Ma Zhenying. Handbook of fungicides [M]. Shanghai: Shanghai Science and Technology Press, 1987, 3:115

5. Huang Yu Yuan. General situation of industrial anti mould (antiseptic) agents [J]. Guangdong chemical industry, 1990, 1:5-10.

6. Bao Yan. Study on analysis method of fungicides in industrial products and environment $[\mathrm{J}]$. Ocean University of China, 2007.

7. Zeng Qifei, Li Shaoguo, Tan Rongxi, et al. Preparation and application of silver nanoparticles [J]. Applied Chemical Engineering, 2014,05:919-922.

8. Wang Xiaoan. Mould prevention in national defense storage [M]. Beijing: National Defense Industry Press, 2001,9:195-225.

9. Wang Chunwei. Preparation and antifungal effect of sodium diacetate compound fungicide $[\mathrm{J}]$. Chinese feed, 2001 (25): 15-16.

10. Lavy,J.J.R. Compbell and T.H. Blackburn: Introductory Microbiology[M], New York, 1993:3445.

11. Dong Yaohua, Guo Na, Liu Tao. Preparation and antibacterial mechanism of nano silver [J]. Journal of Shanghai Maritime University, 2013,34 (01): 80-83.

12.Fayaz A M, Balaji K, Girilal M, et al. Biogenic synthesis of silver nanoparticles and their synergistic effect with antibiotics: a study against gram-positive and gram-negative bacteria[J]. Nanomedicine, 2010,6(1):103-109.

13.Amin R M, Mohamed M B, Ramadan M A, et al. Rapid and sensitive microplate assay for screening the effect of silver and gold nanaparticles on bacteria[J]. Nanomedicine, 2009,4(6):637-643.

14.LI W R, XIE X B, SHI Q S, et al. Antibacterial activity and mechanism of silver nanoparticles on Escherichia coli[J]. Applied Microbiology and Biotechnology, 2010.85(4):1115-1122. 